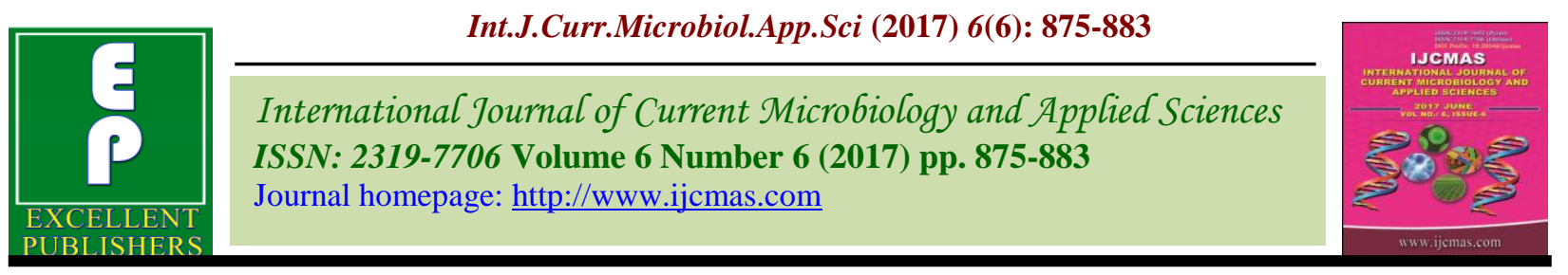

Original Research Article https://doi.org/10.20546/ijcmas.2017.606.103

\title{
Study the Response of Different Phosphorus Levels and Frequency of Boron Levels on Growth and Yield of Greengram
}

\author{
Preeti Choudhary*, Gautam Ghosh, Neha and Shobha Kumari
}

Department of Agronomy, Sam Higginbottom Institute of Agriculture, Technology and Sciences,

(Formerly Allahabad Agricultural Institute), (Deemed to-be-University),

Allahabad - 211007 (U.P), India

*Corresponding author

\section{A B S T R A C T}

Keywords

Mung bean, Phosphorus levels, Boron frequency, Grain and Straw yield.

Article Info

Accepted: 17 May 2017 Available Online: 10 June 2017
A field experiment was conducted during Zaid season, 2015 at the Crop Research Farm, Department of Agronomy, SHIATS, Allahabad (U.P.) to concluded the response of different phosphorus levels and frequency of boron levels on growth and yield of summer Greengram (Vigna radiata L.) in Randomized Block Design with twelve treatments replicated thrice. Among the different phosphorus levels and frequency of boron levels under in treatment T11 i.e., N3(20:60:20 NPK) + $0.2 \%$ foliar spray of borax at 35DAS (pre-flowering) recorded maximum plant height $(53.60 \mathrm{~cm})$, number of leaves plant ${ }^{-1}(21.16)$, number of branches plant ${ }^{-1}$ (6.76), number of nodules plant ${ }^{-1}(8.80)$, dry weight $(24.82 \mathrm{~g})$, crop growth rate $\left(0.53 \mathrm{~g} \mathrm{~m}^{-2}\right.$ day $\left.^{-1}\right)$, relative growth rate $\left(0.04 \mathrm{~g} \mathrm{~g}^{-1}\right.$ day $\left.^{-1}\right)$, number of pods plant ${ }^{-1}$ (42.46), average number of grain pod $^{-1}(13.40)$, pod length $(10.80 \mathrm{~cm})$, test weight $(47.00 \mathrm{~g})$, grain yield $\left(1.62 \mathrm{t} \mathrm{ha}^{-1}\right)$, straw yield $\left(2.85 \mathrm{t} \mathrm{ha}^{-1}\right)$, protein content $(24.56 \%)$ and harvest index $(36.15 \%)$. Whereas the lowest value $(48.26 \mathrm{~cm}, 18.93$ plant $^{-1}, 6.20$ plant $^{-1}, 5.53$ plant $^{-1}, 20.02 \mathrm{~g}, 0.39 \mathrm{~g} \mathrm{~m}^{-2}$ day $^{-1}, 0.03 \mathrm{~g} \mathrm{~g}^{-1}$ day $^{-1}, 30.40$ plant $^{-1}, 7.73$ pod $^{-1}, 8.13 \mathrm{~cm}, 41.06 \mathrm{~g}, 0.99 \mathrm{t} \mathrm{ha}^{-1}, 2.06 \mathrm{t} \mathrm{ha}^{-1}, 20.36 \%$ and $32.58 \%$ respectively) in the treatment T1 i.e., N1 (20:40:20 NPK).

\section{Introduction}

Mung bean [Vigna radiata (L.) Wilczek] is of ancient cultivation in India. In India mung bean is grown in almost all parts of the country. It is grown in India during kharif, but is also grown in spring or summer season in irrigated northern plains as a rabi crop in southern and south-eastern parts where is winter is mild. Mung bean is an excellent source of protein $(25 \%)$ with high quality of lysine $(460 \mathrm{mg} / \mathrm{g})$ and tryptophan $(60 \mathrm{mg} / \mathrm{g})$. It also has remarkable quantity of ascorbic acid when sprouted and also have riboflavin $(0.21 \mathrm{mg} / 100 \mathrm{~g})$ and minerals $(3.84 \mathrm{~g} / 100 \mathrm{~g})$. The total area under pulses is 23.63 mha with an annual production of $14.76 \mathrm{M}$ tonnes in the country. In India green gram occupies 3.4 million hectare area and contributes to 1.4 million tonnes in pulse production (Anonymous. 2010-11). Mungbean contributes $14 \%$ in total pulse area and $7 \%$ in total pulse production in India. The low productivity of mungbean may be due to 
nutritional deficiency in soil and imbalanced external fertilization (Awomi et al., 2012). Phosphorus is an important plant nutrient for greengram. Indian soils are poor to medium in available phosphorus. Only about 30 per cent of the applied phosphorus is available for crops and remaining part converted into insoluble phosphorus (Sharma and Khurana, 1997). Phosphorus fertilization occupies an important place amongst the non-renewable inputs in modern agriculture. Crop recovery of added phosphorus seldom exceeds 20 per cent and it may be improve by the judicious management. As the concentration of available $\mathrm{P}$ in the soil solution is normally insufficient to support the plant growth, continual replacement of soluble $\mathrm{P}$ from inorganic and organic sources is necessary to meet the $\mathrm{P}$ requirements of crop (Tisdale et al., 2010). Additional application of $\mathrm{P}$ is Increase nodule formation which increases nitrogen fixation transduction, macromolecular biosynthesis and respiration. Phosphorus, the master key element is known to be involved in a plethora of functions in the plant growth and metabolism. Among the various factors responsible for maximizing the yield of greengram, phosphorus levels and frequency of boron levels is most important. It is necessary to use them economically in combination with phosphorus and boron, as greengram shows high response to high phosphorus levels and frequency of boron levels.

\section{Materials and Methods}

Field experiment was conducted during Zaid season 2015 at Crop Research Farm, Sam Higginbottom Institute of Agriculture, Technology and Sciences (Deemed-to-beUniversity) Allahabad. The experimental site is located at $25057 \mathrm{~N}$ latitude, $87019 \mathrm{E}$ longitude and at an altitude of above mean sea level. The soil of the experimental area was sandy loam with moderately alkaline $\mathrm{pH}$; low in organic carbon $(0.32 \%)$ and available $\mathrm{N}$
(188.30 kg ha ${ }^{-1}$ ), available $\mathrm{P}\left(34.50 \mathrm{~kg} \mathrm{ha}^{-1}\right.$ ) and available $\mathrm{K}\left(87.00 \mathrm{~kg} \mathrm{ha}^{-1}\right)$ during zaid 2015 respectively. A recommended greengram variety (SAMRAT) was chosen for the study. The experiment was laid out in Randomized Block Design (RBD) with two factor different levels of phosphorus [20:50:20 kg ha ${ }^{-1}, 20: 60: 20 \mathrm{~kg} \mathrm{ha}^{-1}$ (P3)] and frequency levels of boron [no application, 20 and 35 DAS, $0.2 \%$ foliar spray of borax] with twelve treatments combination on a plot size of $3 \times 3 \mathrm{~m}^{2}$. Before sowing, line were formed in the field as the spacing in treatments. Mungbean was sown in line and covered with the soil. Mungbean seeds were hand dibbled. The total quantity of nitrogen, phosphorus and potassium as per treatment in the form of Urea (46\%), single super phosphate (16\%) and MOP (60\%) respectively were applied below the seeds at the time of sowing and $0.2 \%$ solution of borax was prepared and sprayed at 20 and 35 DAS. All the agronomic practices were carried out uniformly to raised the crop. For taking data on yield and yield components on mungbean five plants were selected randomly in each plot.

\section{Results and Discussion}

\section{Numbers of pod plant ${ }^{-1}$}

Numbers of pods plant ${ }^{-1}$ were significantly affected by phosphorus and boron application. Significantly maximum (42.46) numbers of pods plant-1 were obtained in T11 N3 $(20: 60: 20 \mathrm{NPK})+35 \mathrm{DAS}(0.2 \%$ foliar spray of borax). The significant minimum (30.40) numbers of pods plant- 1 were obtained in $\mathrm{T} 1$ N1 (20:40:20 NPK). This might be due to boron helps in pollen formation and grain formation. According to Dutta et al., (1984) numbers of pods per plant are increased by boron application. These results are in line with Kaisher et al., (2010). Numbers of pods per plant were significantly affected by boron application. 


\section{Pod length (cm)}

Phosphorus and boron levels exerted a significant effect pods length. The significantly maximum pods length per plant $(10.80 \mathrm{~cm})$ were produced by application of (20:60:20 NPK) + 35DAS (0.2\% foliar spray of borax) T11 N3. The pod length may be due to application of boron and involved in seed formation thereby increases pods length per plant.

The minimum pods length per plant $(8.13 \mathrm{~cm})$ were found in TI NI (20:40:20 NPK) application. These results are in line with Kaisher et al., (2010). The pods length per plant was significantly affected by phosphorus and boron application. This might be due to boron helps in pollen formation and grain formation. According to Dutta et al., (1984) pods length per plant is increased by boron application.

\section{Number of seeds per pod}

Phosphorus and boron has significant effect on number of seeds per pod. Significantly maximum numbers of seeds per pod (13.40) were found in T11 N3 of (20:60:20 NPK) + 35DAS $(0.2 \%$ foliar spray of borax $)$ application. It might be due to phosphorus and boron which is involved in seed formation and thereby increases number of seeds per pod. Significantly minimum numbers of seeds per pod were found in T1 N1 (20:40:20 NPK) application alone. Mondal et al., (2003) stated that numbers of seeds per pod were significantly increased by application of sulphur and boron in mungbean. It may be due to boron application, which is involved in flower and grain formation and thereby increase number of seeds per pod. Minimum numbers of seeds per pod were found with no boron application, that is application of (20:40:20 NPK) alone. These results are in line with Kaisher et al., (2010).

Table.1 Effect of phosphorus levels and frequency of boron levels on plant height $(\mathrm{cm})$ at different stages of crop growth in greengram

\begin{tabular}{|c|c|c|c|c|c|}
\hline \multirow{2}{*}{ No. } & \multirow{2}{*}{ Treatment } & \multicolumn{4}{|c|}{ Plant height $(\mathrm{cm})$} \\
\hline & & 15 DAS & 30 DAS & 45 DAS & 60 DAS \\
\hline $\mathrm{T}_{1}$ & $\mathrm{~N}_{1}(20: 40: 20 \mathrm{NPK})$ & 10.98 & 20.04 & 41.06 & 48.26 \\
\hline $\mathrm{T}_{2}$ & $\mathrm{~N}_{2}(20: 40: 20 \mathrm{NPK})+20 \mathrm{DAS}(0.2 \% \mathrm{FsB})$ & 12.30 & 20.92 & 41.93 & 50.63 \\
\hline $\mathrm{T}_{3}$ & $\mathrm{~N}_{1}(20: 40: 20 \mathrm{NPK})+35 \mathrm{DAS}(0.2 \% \mathrm{FsB})$ & 11.48 & 21.86 & 43.70 & 48.33 \\
\hline $\mathrm{T}_{4}$ & $\mathrm{~N}_{1}(20: 40: 20 \mathrm{NPK})+20$ and 35 DAS $(0.2 \% \mathrm{FsB})$ & 11.63 & 20.46 & 44.30 & 50.66 \\
\hline $\mathrm{T}_{5}$ & $\mathrm{~N}_{2}(20: 50: 20 \mathrm{NPK})$ & 12.08 & 20.38 & 41.36 & 50.46 \\
\hline $\mathrm{T}_{6}$ & $\mathrm{~N}_{2}(20: 50: 20 \mathrm{NPK})+20 \mathrm{DAS}(0.2 \% \mathrm{FsB})$ & 11.64 & 21.72 & 44.33 & 52.00 \\
\hline $\mathrm{T}_{7}$ & $\mathrm{~N}_{2}(20: 50: 20 \mathrm{NPK})+35 \mathrm{DAS}(0.2 \% \mathrm{FsB})$ & 13.00 & 24.20 & 46.86 & 52.66 \\
\hline $\mathrm{T}_{8}$ & $\mathrm{~N} 2(20: 50: 20 \mathrm{NPK})+20$ and $35 \mathrm{DAS}(0.2 \% \mathrm{FsB})$ & 12.36 & 20.61 & 44.66 & 51.53 \\
\hline $\mathrm{T}_{9}$ & $\mathrm{~N}_{3}(20: 60: 20 \mathrm{NPK})$ & 12.73 & 20.62 & 43.86 & 50.83 \\
\hline $\mathrm{T}_{10}$ & $\mathrm{~N}_{3}(20: 60: 20 \mathrm{NPK})+20 \mathrm{DAS}(0.2 \% \mathrm{FsB})$ & 12.27 & 20.59 & 43.80 & 52.66 \\
\hline $\mathrm{T}_{11}$ & $\mathrm{~N}_{3}(20: 60: 20 \mathrm{NPK})+35 \mathrm{DAS}(0.2 \% \mathrm{FsB})$ & 12.96 & 24.82 & 47.46 & 53.60 \\
\hline \multirow[t]{4}{*}{$\mathrm{T}_{12}$} & $\mathrm{~N}_{3}(20: 60: 20 \mathrm{NPK})+20$ and 35 DAS $(0.2 \% \mathrm{FsB})$ & 12.54 & 20.46 & 45.66 & 51.30 \\
\hline & F- test & NS & NS & $\mathrm{S}$ & $\mathrm{S}$ \\
\hline & S. Ed. $( \pm)$ & 1.12 & 1.37 & 1.53 & 1.31 \\
\hline & C. D. $(P=0.05)$ & - & - & 3.18 & 2.72 \\
\hline
\end{tabular}

FsB -Foliar spray of Boron 
Table.2 Effect of phosphorus levels and frequency of boron levels on number of leaves plant ${ }^{-1}$ at different stages of crop growth in greengram

\begin{tabular}{|c|c|c|c|c|c|}
\hline \multirow{2}{*}{ No. } & \multirow{2}{*}{ Treatment } & \multicolumn{4}{|c|}{ Leaves plant $^{-1}$} \\
\hline & & 15 DAS & 30 DAS & 45 DAS & 60 DAS \\
\hline $\mathrm{T}_{1}$ & $\mathrm{~N}_{1}(20: 40: 20 \mathrm{NPK})$ & 2.66 & 13.40 & 22.00 & 18.93 \\
\hline $\mathrm{T}_{2}$ & $\mathrm{~N}_{2}(20: 40: 20 \mathrm{NPK})+20 \mathrm{DAS}(0.2 \% \mathrm{FsB})$ & 3.13 & 15.80 & 22.20 & 20.03 \\
\hline $\mathrm{T}_{3}$ & $\mathrm{~N}_{1}(20: 40: 20 \mathrm{NPK})+35 \mathrm{DAS}(0.2 \% \mathrm{FsB})$ & 3.20 & 16.20 & 22.20 & 19.36 \\
\hline $\mathrm{T}_{4}$ & $\mathrm{~N}_{1}(20: 40: 20 \mathrm{NPK})+20$ and $35 \mathrm{DAS}(0.2 \% \mathrm{FsB})$ & 3.00 & 14.60 & 22.60 & 19.53 \\
\hline $\mathrm{T}_{5}$ & $\mathrm{~N}_{2}(20: 50: 20 \mathrm{NPK})$ & 2.86 & 15.20 & 22.40 & 19.40 \\
\hline $\mathrm{T}_{6}$ & $\mathrm{~N}_{2}(20: 50: 20 \mathrm{NPK})+20 \mathrm{DAS}(0.2 \% \mathrm{FsB})$ & 3.13 & 15.20 & 22.00 & 19.10 \\
\hline $\mathrm{T}_{7}$ & $\mathrm{~N}_{2}(20: 50: 20 \mathrm{NPK})+35 \mathrm{DAS}(0.2 \% \mathrm{FsB})$ & 3.26 & 17.60 & 22.20 & 19.80 \\
\hline $\mathrm{T}_{8}$ & $\mathrm{~N} 2(20: 50: 20 \mathrm{NPK})+20$ and $35 \mathrm{DAS}(0.2 \% \mathrm{FsB})$ & 2.93 & 15.40 & 22.20 & 19.06 \\
\hline $\mathrm{T}_{9}$ & $\mathrm{~N}_{3}(20: 60: 20 \mathrm{NPK})$ & 2.93 & 15.40 & 22.20 & 19.43 \\
\hline $\mathrm{T}_{10}$ & $\mathrm{~N}_{3}(20: 60: 20 \mathrm{NPK})+20 \mathrm{DAS}(0.2 \% \mathrm{FsB})$ & 2.86 & 16.40 & 22.20 & 19.40 \\
\hline $\mathrm{T}_{11}$ & $\mathrm{~N}_{3}(20: 60: 20 \mathrm{NPK})+35 \mathrm{DAS}(0.2 \% \mathrm{FsB})$ & 3.80 & 17.80 & 23.20 & 21.16 \\
\hline \multirow[t]{4}{*}{$\mathrm{T}_{12}$} & $\mathrm{~N}_{3}(20: 60: 20 \mathrm{NPK})+20$ and $35 \mathrm{DAS}(0.2 \% \mathrm{FsB})$ & 3.13 & 14.60 & 22.40 & 19.70 \\
\hline & F- test & NS & NS & NS & $\mathrm{S}$ \\
\hline & S. Ed. $( \pm)$ & 0.26 & 1.33 & 0.39 & 0.49 \\
\hline & C. D. $(P=0.05)$ & - & - & - & 1.02 \\
\hline
\end{tabular}

FsB -Foliar spray of Boron

Table.3 Effect of phosphorus levels and frequency of boron levels on number of branches plant $^{-1}$ at different stages of crop growth in greengram

\begin{tabular}{|c|c|c|c|c|}
\hline \multirow[b]{2}{*}{ No. } & \multirow[t]{2}{*}{ Treatment } & \multicolumn{3}{|c|}{ No. of branches plant ${ }^{-1}$} \\
\hline & & $\begin{array}{c}\text { 30 } \\
\text { DAS }\end{array}$ & 45DAS & 60 DAS \\
\hline $\mathrm{T}_{1}$ & $\mathrm{~N}_{1}(20: 40: 20 \mathrm{NPK})$ & 0.20 & 5.46 & 6.20 \\
\hline $\mathrm{T}_{2}$ & $\mathrm{~N}_{2}(20: 40: 20 \mathrm{NPK})+20 \mathrm{DAS}(0.2 \% \mathrm{FsB})$ & 0.40 & 5.60 & 6.36 \\
\hline $\mathrm{T}_{3}$ & $\mathrm{~N}_{1}(20: 40: 20 \mathrm{NPK})+35 \mathrm{DAS}(0.2 \% \mathrm{FsB})$ & 0.46 & 5.66 & 6.33 \\
\hline $\mathrm{T}_{4}$ & $\mathrm{~N}_{1}(20: 40: 20 \mathrm{NPK})+20$ and $35 \mathrm{DAS}(0.2 \% \mathrm{FsB})$ & 0.80 & 5.66 & 6.53 \\
\hline $\mathrm{T}_{5}$ & $\mathrm{~N}_{2}(20: 50: 20 \mathrm{NPK})$ & 0.60 & 5.66 & 6.30 \\
\hline $\mathrm{T}_{6}$ & $\mathrm{~N}_{2}(20: 50: 20 \mathrm{NPK})+20 \mathrm{DAS}(0.2 \% \mathrm{FsB})$ & 0.66 & 5.53 & 6.53 \\
\hline $\mathrm{T}_{7}$ & $\mathrm{~N}_{2}(20: 50: 20 \mathrm{NPK})+35 \mathrm{DAS}(0.2 \% \mathrm{FsB})$ & 0.66 & 5.60 & 6.53 \\
\hline $\mathrm{T}_{8}$ & $\mathrm{~N} 2(20: 50: 20 \mathrm{NPK})+20$ and $35 \mathrm{DAS}(0.2 \% \mathrm{FsB})$ & 0.33 & 5.53 & 6.36 \\
\hline $\mathrm{T}_{9}$ & $\mathrm{~N}_{3}(20: 60: 20 \mathrm{NPK})$ & 0.33 & 5.60 & 6.70 \\
\hline $\mathrm{T}_{10}$ & $\mathrm{~N}_{3}(20: 60: 20 \mathrm{NPK})+20 \mathrm{DAS}(0.2 \% \mathrm{FsB})$ & 0.33 & 5.60 & 6.73 \\
\hline $\mathrm{T}_{11}$ & $\mathrm{~N}_{3}(20: 60: 20 \mathrm{NPK})+35 \mathrm{DAS}(0.2 \% \mathrm{FsB})$ & 0.80 & 5.73 & 6.76 \\
\hline \multirow[t]{4}{*}{$\mathrm{T}_{12}$} & $\mathrm{~N}_{3}(20: 60: 20 \mathrm{NPK})+20$ and $35 \mathrm{DAS}(0.2 \% \mathrm{FsB})$ & 0.73 & 5.66 & 6.36 \\
\hline & F- test & NS & NS & $\mathrm{S}$ \\
\hline & S. Ed. $( \pm)$ & 0.33 & 0.13 & 0.16 \\
\hline & C. D. $(P=0.05)$ & - & - & 0.33 \\
\hline
\end{tabular}

FsB -Foliar spray of Boron 
Table.4 Effect of phosphorus levels and frequency of boron levels on number of nodules plant ${ }^{-1}$ at different stages of crop growth in greengram

\begin{tabular}{|c|c|c|c|c|}
\hline \multirow{2}{*}{ No. } & \multirow[t]{2}{*}{ Treatment } & \multicolumn{3}{|c|}{ Number of nodules plant ${ }^{-1}$} \\
\hline & & $\begin{array}{c}30 \\
\text { DAS }\end{array}$ & 45DAS & 60 DAS \\
\hline $\mathrm{T}_{1}$ & $\mathrm{~N}_{1}(20: 40: 20 \mathrm{NPK})$ & 8.63 & 26.00 & 5.53 \\
\hline $\mathrm{T}_{2}$ & $\mathrm{~N}_{2}(20: 40: 20 \mathrm{NPK})+20 \mathrm{DAS}(0.2 \% \mathrm{FsB})$ & 8.73 & 28.60 & 6.40 \\
\hline $\mathrm{T}_{3}$ & $\mathrm{~N}_{1}(20: 40: 20 \mathrm{NPK})+35 \mathrm{DAS}(0.2 \% \mathrm{FsB})$ & 8.83 & 28.26 & 6.46 \\
\hline $\mathrm{T}_{4}$ & $\mathrm{~N}_{1}(20: 40: 20 \mathrm{NPK})+20$ and $35 \mathrm{DAS}(0.2 \% \mathrm{FsB})$ & 9.40 & 27.13 & 6.66 \\
\hline $\mathrm{T}_{5}$ & $\mathrm{~N}_{2}(20: 50: 20 \mathrm{NPK})$ & 10.13 & 28.66 & 6.00 \\
\hline $\mathrm{T}_{6}$ & $\mathrm{~N}_{2}(20: 50: 20 \mathrm{NPK})+20 \mathrm{DAS}(0.2 \% \mathrm{FsB})$ & 8.96 & 28.20 & 6.86 \\
\hline $\mathrm{T}_{7}$ & $\mathrm{~N}_{2}(20: 50: 20 \mathrm{NPK})+35 \mathrm{DAS}(0.2 \% \mathrm{FsB})$ & 9.60 & 30.26 & 7.40 \\
\hline $\mathrm{T}_{8}$ & $\mathrm{~N} 2(20: 50: 20 \mathrm{NPK})+20$ and $35 \mathrm{DAS}(0.2 \% \mathrm{FsB})$ & 9.00 & 29.40 & 5.66 \\
\hline $\mathrm{T}_{9}$ & $\mathrm{~N}_{3}(20: 60: 20 \mathrm{NPK})$ & 8.80 & 28.13 & 6.13 \\
\hline $\mathrm{T}_{10}$ & $\mathrm{~N}_{3}(20: 60: 20 \mathrm{NPK})+20 \mathrm{DAS}(0.2 \% \mathrm{FsB})$ & 9.20 & 28.80 & 6.80 \\
\hline $\mathrm{T}_{11}$ & $\mathrm{~N}_{3}(20: 60: 20 \mathrm{NPK})+35$ DAS $(0.2 \% \mathrm{FsB})$ & 10.86 & 32.26 & 8.80 \\
\hline \multirow[t]{4}{*}{$\mathrm{T}_{12}$} & $\mathrm{~N}_{3}(20: 60: 20 \mathrm{NPK})+20$ and $35 \mathrm{DAS}(0.2 \% \mathrm{FsB})$ & 9.13 & 28.00 & 6.20 \\
\hline & F- test & NS & $\mathrm{S}$ & $\mathrm{S}$ \\
\hline & S. Ed. $( \pm)$ & 0.57 & 1.10 & 0.59 \\
\hline & C. D. $(P=0.05)$ & - & 2.29 & 1.21 \\
\hline
\end{tabular}

FsB -Foliar spray of Boron

Table.5 Effect of phosphorus levels and frequency of boron levels on dry weight ( $\mathrm{g}$ ) at different stages of crop growth in greengram

\begin{tabular}{|c|c|c|c|c|c|}
\hline \multirow[b]{2}{*}{ No. } & \multirow[t]{2}{*}{ Treatment } & \multicolumn{4}{|c|}{ Dry weight (g) } \\
\hline & & $\begin{array}{c}15 \\
\text { DAS }\end{array}$ & 30 DAS & 45 DAS & $\begin{array}{c}60 \\
\text { DAS }\end{array}$ \\
\hline $\mathrm{T}_{1}$ & $\mathrm{~N}_{1}(20: 40: 20 \mathrm{NPK})$ & 0.23 & 1.45 & 7.20 & 20.04 \\
\hline $\mathrm{T}_{2}$ & $\mathrm{~N}_{2}(20: 40: 20 \mathrm{NPK})+20 \mathrm{DAS}(0.2 \% \mathrm{FsB})$ & 0.24 & 1.58 & 8.30 & 21.34 \\
\hline $\mathrm{T}_{3}$ & $\mathrm{~N}_{1}(20: 40: 20 \mathrm{NPK})+35 \mathrm{DAS}(0.2 \% \mathrm{FsB})$ & 0.33 & 1.75 & 8.16 & 21.43 \\
\hline $\mathrm{T}_{4}$ & $\mathrm{~N}_{1}(20: 40: 20 \mathrm{NPK})+20$ and 35 DAS $(0.2 \% \mathrm{FsB})$ & 0.28 & 1.55 & 8.41 & 20.46 \\
\hline $\mathrm{T}_{5}$ & $\mathrm{~N}_{2}(20: 50: 20 \mathrm{NPK})$ & 0.34 & 1.83 & 9.00 & 20.59 \\
\hline $\mathrm{T}_{6}$ & $\mathrm{~N}_{2}(20: 50: 20 \mathrm{NPK})+20 \mathrm{DAS}(0.2 \% \mathrm{FsB})$ & 0.27 & 1.65 & 7.91 & 21.72 \\
\hline $\mathrm{T}_{7}$ & $\mathrm{~N}_{2}(20: 50: 20 \mathrm{NPK})+35 \mathrm{DAS}(0.2 \% \mathrm{FsB})$ & 0.42 & 1.50 & 9.25 & 24.20 \\
\hline $\mathrm{T}_{8}$ & $\mathrm{~N} 2(20: 50: 20 \mathrm{NPK})+20$ and $35 \mathrm{DAS}(0.2 \% \mathrm{FsB})$ & 0.36 & 1.58 & 8.11 & 20.61 \\
\hline $\mathrm{T}_{9}$ & $\mathrm{~N}_{3}(20: 60: 20 \mathrm{NPK})$ & 0.30 & 1.58 & 8.71 & 20.62 \\
\hline $\mathrm{T}_{10}$ & $\mathrm{~N}_{3}(20: 60: 20 \mathrm{NPK})+20 \mathrm{DAS}(0.2 \% \mathrm{FsB})$ & 0.33 & 1.86 & 7.86 & 20.38 \\
\hline $\mathrm{T}_{11}$ & $\mathrm{~N}_{3}(20: 60: 20 \mathrm{NPK})+35$ DAS $(0.2 \% \mathrm{FsB})$ & 0.36 & 1.90 & 10.03 & 24.82 \\
\hline \multirow[t]{4}{*}{$\mathrm{T}_{12}$} & $\mathrm{~N}_{3}(20: 60: 20 \mathrm{NPK})+20$ and 35 DAS $(0.2 \% \mathrm{FsB})$ & 0.24 & 1.48 & 8.10 & 20.46 \\
\hline & F- test & NS & NS & $\mathrm{S}$ & $\mathrm{S}$ \\
\hline & S. Ed. $( \pm)$ & 0.08 & 0.22 & 0.58 & 1.30 \\
\hline & C. D. $(P=0.05)$ & - & - & 1.19 & 2.69 \\
\hline
\end{tabular}


Table.6 Effect of phosphorus levels and frequency of boron levels on plant crop growth rate ( $\mathrm{g}$ $\mathrm{m}^{-2}$ day ${ }^{-1}$ ) at different stages of crop growth in greengram

\begin{tabular}{|c|c|c|c|c|c|}
\hline \multirow{3}{*}{ No. } & \multirow[t]{3}{*}{ Treatment } & \multicolumn{4}{|c|}{ Crop growth rate $\left(\mathrm{g} \mathrm{m}^{-2}\right.$ day $\left.^{-1}\right)$} \\
\hline & & $0-15$ & $15-30$ & $30-45$ & $45-60$ \\
\hline & & DAS & DAS & DAS & DAS \\
\hline $\mathrm{T}_{1}$ & $\mathrm{~N}_{1}(20: 40: 20 \mathrm{NPK})$ & 0.01 & 0.06 & 0.38 & 0.39 \\
\hline $\mathrm{T}_{2}$ & $\mathrm{~N}_{2}(20: 40: 20 \mathrm{NPK})+20 \mathrm{DAS}(0.2 \% \mathrm{FsB})$ & 0.01 & 0.08 & 0.44 & 0.51 \\
\hline $\mathrm{T}_{3}$ & $\mathrm{~N}_{1}(20: 40: 20 \mathrm{NPK})+35 \mathrm{DAS}(0.2 \% \mathrm{FsB})$ & 0.02 & 0.09 & 0.45 & 0.46 \\
\hline $\mathrm{T}_{4}$ & $\mathrm{~N}_{1}(20: 40: 20 \mathrm{NPK})+20$ and $35 \mathrm{DAS}(0.2 \% \mathrm{FsB})$ & 0.01 & 0.08 & 0.45 & 0.52 \\
\hline $\mathrm{T}_{5}$ & $\mathrm{~N}_{2}(20: 50: 20 \mathrm{NPK})$ & 0.01 & 0.09 & 0.47 & 0.43 \\
\hline $\mathrm{T}_{6}$ & $\mathrm{~N}_{2}(20: 50: 20 \mathrm{NPK})+20 \mathrm{DAS}(0.2 \% \mathrm{FsB})$ & 0.01 & 0.09 & 0.41 & 0.51 \\
\hline $\mathrm{T}_{7}$ & $\mathrm{~N}_{2}(20: 50: 20 \mathrm{NPK})+35 \mathrm{DAS}(0.2 \% \mathrm{FsB})$ & 0.02 & 0.08 & 0.49 & 0.43 \\
\hline $\mathrm{T}_{8}$ & $\mathrm{~N} 2(20: 50: 20 \mathrm{NPK})+20$ and $35 \mathrm{DAS}(0.2 \% \mathrm{FsB})$ & 0.02 & 0.08 & 0.43 & 0.50 \\
\hline $\mathrm{T}_{9}$ & $\mathrm{~N}_{3}(20: 60: 20 \mathrm{NPK})$ & 0.02 & 0.08 & 0.47 & 0.47 \\
\hline $\mathrm{T}_{10}$ & $\mathrm{~N}_{3}(20: 60: 20 \mathrm{NPK})+20 \mathrm{DAS}(0.2 \% \mathrm{FsB})$ & 0.02 & 0.09 & 0.39 & 0.51 \\
\hline $\mathrm{T}_{11}$ & $\mathrm{~N}_{3}(20: 60: 20 \mathrm{NPK})+35$ DAS $(0.2 \% \mathrm{FsB})$ & 0.07 & 0.10 & 0.50 & 0.53 \\
\hline \multirow[t]{4}{*}{$\mathrm{T}_{12}$} & $\mathrm{~N}_{3}(20: 60: 20 \mathrm{NPK})+20$ and $35 \mathrm{DAS}(0.2 \% \mathrm{FsB})$ & 0.01 & 0.08 & 0.43 & 0.52 \\
\hline & F- test & NS & NS & NS & NS \\
\hline & S. Ed. $( \pm)$ & 0.02 & 0.02 & 0.06 & 0.05 \\
\hline & C. D. $(P=0.05)$ & - & - & - & - \\
\hline
\end{tabular}

FsB -Foliar spray of Boron

Table.7 Effect of phosphorus levels and frequency of boron levels on relative growth rate $\left(\mathrm{g} \mathrm{g}^{-1 \text { day-1 }}\right)$ at different stages of crop growth in greengram

\begin{tabular}{|c|c|c|c|c|c|}
\hline \multirow{3}{*}{ No. } & \multirow[t]{3}{*}{ Treatment } & \multicolumn{4}{|c|}{ Relative Growth Rate $\left(\mathrm{g} \mathrm{g}^{-1}\right.$ day $\left.^{-1}\right)$} \\
\hline & & $0-15$ & $15-30$ & $30-45$ & 45-60 \\
\hline & & DAS & DAS & DAS & DAS \\
\hline $\mathrm{T}_{1}$ & $\mathrm{~N}_{1}(20: 40: 20 \mathrm{NPK})$ & 0.06 & 0.08 & 0.09 & 0.03 \\
\hline $\mathrm{T}_{2}$ & $\mathrm{~N}_{2}(20: 40: 20 \mathrm{NPK})+20$ DAS $(0.2 \% \mathrm{FsB})$ & 0.09 & 0.12 & 0.11 & 0.04 \\
\hline $\mathrm{T}_{3}$ & $\mathrm{~N}_{1}(20: 40: 20 \mathrm{NPK})+35 \mathrm{DAS}(0.2 \% \mathrm{FsB})$ & 0.07 & 0.11 & 0.10 & 0.04 \\
\hline $\mathrm{T}_{4}$ & $\mathrm{~N}_{1}(20: 40: 20 \mathrm{NPK})+20$ and 35 DAS $(0.2 \% \mathrm{FsB})$ & 0.08 & 0.11 & 0.11 & 0.03 \\
\hline $\mathrm{T}_{5}$ & $\mathrm{~N}_{2}(20: 50: 20 \mathrm{NPK})$ & 0.07 & 0.10 & 0.10 & 0.03 \\
\hline $\mathrm{T}_{6}$ & $\mathrm{~N}_{2}(20: 50: 20 \mathrm{NPK})+20 \mathrm{DAS}(0.2 \% \mathrm{FsB})$ & 0.09 & 0.12 & 0.10 & 0.04 \\
\hline $\mathrm{T}_{7}$ & $\mathrm{~N}_{2}(20: 50: 20 \mathrm{NPK})+35 \mathrm{DAS}(0.2 \% \mathrm{FsB})$ & 0.09 & 0.12 & 0.12 & 0.03 \\
\hline $\mathrm{T}_{8}$ & $\mathrm{~N} 2(20: 50: 20 \mathrm{NPK})+20$ and $35 \mathrm{DAS}(0.2 \% \mathrm{FsB})$ & 0.06 & 0.09 & 0.11 & 0.04 \\
\hline $\mathrm{T}_{9}$ & $\mathrm{~N}_{3}(20: 60: 20 \mathrm{NPK})$ & 0.07 & 0.10 & 0.11 & 0.03 \\
\hline $\mathrm{T}_{10}$ & $\mathrm{~N}_{3}(20: 60: 20 \mathrm{NPK})+20$ DAS $(0.2 \% \mathrm{FsB})$ & 0.07 & 0.11 & 0.10 & 0.04 \\
\hline $\mathrm{T}_{11}$ & $\mathrm{~N}_{3}(20: 60: 20 \mathrm{NPK})+35$ DAS $(0.2 \% \mathrm{FsB})$ & 0.10 & 0.68 & 0.39 & 0.04 \\
\hline \multirow[t]{4}{*}{$\mathrm{T}_{12}$} & $\mathrm{~N}_{3}(20: 60: 20 \mathrm{NPK})+20$ and 35 DAS $(0.2 \% \mathrm{FsB})$ & 0.09 & 0.12 & 0.11 & 0.04 \\
\hline & F- test & NS & NS & NS & NS \\
\hline & S. Ed. $( \pm)$ & 0.02 & 0.24 & 0.11 & 0.01 \\
\hline & C. D. $(P=0.05)$ & - & - & - & - \\
\hline
\end{tabular}

FsB -Foliar spray of Boron 


\section{Int.J.Curr.Microbiol.App.Sci (2017) 6(6): 875-883}

Table.8 Effect of different levels of phosphorus and frequency of boron levels on yield and yield attributes of Greengram

\begin{tabular}{|c|c|c|c|c|c|c|c|c|}
\hline & Treatment & $\begin{array}{l}\text { No. of pods } \\
\text { plant }^{-1}\end{array}$ & $\begin{array}{l}\text { Pod length } \\
(\mathrm{cm})\end{array}$ & $\begin{array}{l}\text { No. of grains } \\
\text { pod }^{-1}\end{array}$ & $\begin{array}{l}\text { test } \\
\text { weight } \\
\text { (g) }\end{array}$ & $\begin{array}{l}\text { Grain } \\
\text { yield } \\
\left(\mathrm{t} \mathrm{ha}^{-1}\right)\end{array}$ & $\begin{array}{l}\text { Straw yield (t } \\
\left.\text { ha }^{-1}\right)\end{array}$ & $\begin{array}{l}\text { Harvest } \\
\text { index }(\%)\end{array}$ \\
\hline $\mathrm{T}_{1}$ & $\mathrm{~N}_{1}(20: 40: 20 \mathrm{NPK})$ & 30.40 & 8.13 & 7.73 & 41.06 & 0.99 & 2.06 & 32.58 \\
\hline $\mathrm{T}_{2}$ & $\mathrm{~N}_{2}(20: 40: 20 \mathrm{NPK})+20 \mathrm{DAS}(0.2 \% \mathrm{FsB})$ & 31.00 & 8.46 & 7.73 & 41.86 & 1.12 & 2.28 & 32.93 \\
\hline $\mathrm{T}_{3}$ & $\mathrm{~N}_{1}(20: 40: 20 \mathrm{NPK})+35 \mathrm{DAS}(0.2 \% \mathrm{FsB})$ & 37.40 & 9.66 & 11.20 & 45.13 & 1.22 & 2.33 & 34.90 \\
\hline $\mathrm{T}_{4}$ & $\begin{array}{l}\mathrm{N}_{1}(20: 40: 20 \mathrm{NPK})+20 \text { and } 35 \text { DAS }(0.2 \% \\
\text { FsB })\end{array}$ & 32.06 & 9.80 & 10.13 & 43.86 & 1.28 & 2.38 & 34.83 \\
\hline $\mathrm{T}_{5}$ & $\mathrm{~N}_{2}(20: 50: 20 \mathrm{NPK})$ & 34.06 & 8.66 & 11.06 & 42.86 & 1.22 & 2.33 & 35.74 \\
\hline $\mathrm{T}_{6}$ & $\mathrm{~N}_{2}(20: 50: 20 \mathrm{NPK})+20 \mathrm{DAS}(0.2 \% \mathrm{FsB})$ & 36.86 & 9.66 & 11.40 & 43.46 & 1.34 & 2.46 & 34.36 \\
\hline $\mathrm{T}_{7}$ & $\mathrm{~N}_{2}(20: 50: 20 \mathrm{NPK})+35 \mathrm{DAS}(0.2 \% \mathrm{FsB})$ & 40.40 & 10.33 & 12.60 & 45.73 & 1.36 & 2.67 & 33.82 \\
\hline $\mathrm{T}_{8}$ & $\begin{array}{l}\mathrm{N} 2(20: 50: 20 \mathrm{NPK})+20 \text { and } 35 \mathrm{DAS}(0.2 \% \\
\text { FsB })\end{array}$ & 35.00 & 10.20 & 11.53 & 43.26 & 1.33 & 2.67 & 33.30 \\
\hline $\mathrm{T}_{9}$ & $\mathrm{~N}_{3}(20: 60: 20 \mathrm{NPK})$ & 31.73 & 9.86 & 11.46 & 43.60 & 1.24 & 2.43 & 35.08 \\
\hline $\mathrm{T}_{10}$ & $\mathrm{~N}_{3}(20: 60: 20 \mathrm{NPK})+20 \mathrm{DAS}(0.2 \% \mathrm{FsB})$ & 38.80 & 10.33 & 11.66 & 44.60 & 1.35 & 2.48 & 35.24 \\
\hline $\mathrm{T}_{11}$ & $\mathrm{~N}_{3}(20: 60: 20 \mathrm{NPK})+35 \mathrm{DAS}(0.2 \% \mathrm{FsB})$ & 42.46 & 10.80 & 13.40 & 47.00 & 1.62 & 2.85 & 36.15 \\
\hline $\mathrm{T}_{12}$ & $\mathrm{~N}_{3}(20: 60: 20 \mathrm{NPK})+20$ and $35 \mathrm{DAS}(0.2 \% \mathrm{FsB})$ & 33.93 & 9.86 & 10.60 & 43.06 & 1.40 & 2.69 & 33.99 \\
\hline & F- test & $\mathrm{S}$ & $\mathrm{S}$ & $\mathrm{S}$ & $\mathrm{S}$ & $\mathrm{S}$ & NS & $\mathrm{S}$ \\
\hline & S. Ed. $( \pm)$ & 1.43 & 0.58 & 0.47 & 0.61 & 0.04 & 0.62 & 0.73 \\
\hline & C. D. $(P=0.05)$ & 2.96 & 1.21 & 0.98 & 1.26 & 0.09 & - & 1.52 \\
\hline
\end{tabular}


Table.9 Effect of phosphorus levels and frequency of boron levels on protein content (\%)

\begin{tabular}{cll}
\hline No. & \multicolumn{1}{c}{ Treatment } & Protein content (\%) \\
\hline $\mathrm{T}_{1}$ & $\mathrm{~N}_{1}(20: 40: 20 \mathrm{NPK})$ & 20.36 \\
$\mathrm{~T}_{2}$ & $\mathrm{~N}_{2}(20: 40: 20 \mathrm{NPK})+20 \mathrm{DAS}(0.2 \% \mathrm{FsB})$ & 20.70 \\
$\mathrm{~T}_{3}$ & $\mathrm{~N}_{1}(20: 40: 20 \mathrm{NPK})+35 \mathrm{DAS}(0.2 \% \mathrm{FsB})$ & 21.43 \\
$\mathrm{~T}_{4}$ & $\mathrm{~N}_{1}(20: 40: 20 \mathrm{NPK})+20$ and 35 DAS $(0.2 \% \mathrm{FsB})$ & 22.20 \\
$\mathrm{~T}_{5}$ & $\mathrm{~N}_{2}(20: 50: 20 \mathrm{NPK})$ & 22.68 \\
$\mathrm{~T}_{6}$ & $\mathrm{~N}_{2}(20: 50: 20 \mathrm{NPK})+20 \mathrm{DAS}(0.2 \% \mathrm{FsB})$ & 23.07 \\
$\mathrm{~T}_{7}$ & $\mathrm{~N}_{2}(20: 50: 20 \mathrm{NPK})+35 \mathrm{DAS}(0.2 \% \mathrm{FsB})$ & 23.42 \\
$\mathrm{~T}_{8}$ & $\mathrm{~N}_{2}(20: 50: 20 \mathrm{NPK})+20$ and 35 DAS $(0.2 \% \mathrm{FsB})$ & 23.95 \\
$\mathrm{~T}_{9}$ & $\mathrm{~N}_{3}(20: 60: 20 \mathrm{NPK})$ & 22.60 \\
$\mathrm{~T}_{10}$ & $\mathrm{~N}_{3}(20: 60: 20 \mathrm{NPK})+20 \mathrm{DAS}(0.2 \% \mathrm{FsB})$ & 23.23 \\
$\mathrm{~T}_{11}$ & $\mathrm{~N}_{3}(20: 60: 20 \mathrm{NPK})+35 \mathrm{DAS}(0.2 \% \mathrm{FsB})$ & 24.56 \\
$\mathrm{~T}_{12}$ & $\mathrm{~N}_{3}(20: 60: 20 \mathrm{NPK})+20$ and 35 DAS $(0.2 \% \mathrm{FsB})$ & 22.91 \\
\hline
\end{tabular}

FsB -Foliar spray of Boron

\section{0-seed weight}

Phosphorus and boron significantly affected 1000 -seed weight. Highest $(47.00 \mathrm{~g})$ and lowest $(0.99 \mathrm{~g}) 1000$-seed weight was found with T11 N3 of (20:60:20 NPK) + 35DAS ( $0.2 \%$ foliar spray of borax) application then no boron application, respectively. The increase in 1000- seed weight may be due to boron application, which increases the seed weight and size. Singh and Yadav (1997) stated boron significantly increased the 1000seed weight in mungbean. 1000-seed weight was highly affected by the application of different levels of boron. The increase in 1000 -seed weight may be due to boron, which increases the seed weight and size. Minimum 1000 -seed weight was found the application of (20:40:20 NPK) alone. The increase in 1000 -seed weight might be due to positive effect of boron on seed weight and size. These results are in line with Kaisher et al., (2010).

\section{Grain yield}

Grain yield differed significantly due to boron application. The highest grain yield $\left(2.85 \mathrm{tha}^{-1}\right)$ was found in T11 N3 (20:60:20 NPK) + 35 DAS ( $0.2 \%$ foliar spray of boron) application
(Table 1). Boron application significantly increased the seed yield Mondal et al., (2003). Boron has significant effect on seed yield. Highest seed yield $\left(2.85 \mathrm{t} \mathrm{ha}^{-1}\right)$ was found in T11 N3 (20:60:20 NPK) + 35 DAS (0.2\% foliar spray of boron) application. The minimum yield was found with no boron application that is T1 N1 (20:40:20 NPK) alone. These results are almost similar to Saha et al., (1996).

\section{Biological yield}

Foliar spray of boron showed that, there is non-significant effect among the treatments on biological yield of mungbean. Highest biological yield $\left(2.85 \mathrm{t} \mathrm{ha}^{-1}\right)$ was found with T11 N3 (20:60:20 NPK) + 35 DAS (0.2\% foliar spray of boron) application. Increase in biological yield may be due to foliar spray of boron, which significantly enhances straw and grain yield in mungbean. Boron showed significantly maximum biological yield (2.06 $\mathrm{t} \mathrm{ha}^{-1}$ ) with no application of boron that is $\mathrm{T} 1$ N1 (20:40:20) alone. These results are in line with Kaisher et al., (2010).

Among the different phosphorus levels and frequency of boron levels under in treatment 
$\mathrm{T} 11$ i.e., $\mathrm{N} 3(20: 60: 20 \mathrm{NPK})+0.2 \%$ foliar spray of borax at 35DAS (pre-flowering) recorded maximum plant height $(53.60 \mathrm{~cm})$, number of leaves plant $^{-1}$ (21.16) (Table 2), number of branches plant ${ }^{-1}$ (6.76) (Table 3), number of nodules plant ${ }^{-1}$ (8.80) (Table 4), dry weight $(24.82 \mathrm{~g})$ (Table 5), crop growth rate $\left(0.53 \mathrm{~g} \mathrm{~m}^{-2}\right.$ day $\left.^{-1}\right)$ (Table 6), relative growth rate $\left(0.04 \mathrm{~g} \mathrm{~g}^{-1}\right.$ day $\left.^{-1}\right)$ (Table 7), number of pods plant ${ }^{-1}$ (42.46) (Table 8), average number of grain pod $^{-1}(13.40)$, pod length $(10.80 \mathrm{~cm})$, test weight $(47.00 \mathrm{~g})$, grain yield $\left(1.62 \mathrm{t} \mathrm{ha}^{-1}\right)$, straw yield $\left(2.85 \mathrm{t} \mathrm{ha}^{-1}\right)$, protein content $(24.56 \%)$ (Table 9) and harvest index $(36.15 \%)$. Whereas the lowest value $\left(48.26 \mathrm{~cm}, 18.93\right.$ plant $^{-1}, 6.20$ plant $^{-1}$, 5.53 plant $^{-1}, 20.02 \mathrm{~g}, 0.39 \mathrm{~g} \mathrm{~m}^{-2}$ day $^{-1}, 0.03 \mathrm{~g}$ $\mathrm{g}^{-1}$ day $^{-1}, 30.40$ plant $^{-1}, 7.73$ pod $^{-1}, 8.13 \mathrm{~cm}$, $41.06 \mathrm{~g}, 0.99 \mathrm{t} \mathrm{ha}^{-1}, 2.06 \mathrm{t} \mathrm{ha}^{-1}, 20.36 \%$ and $32.58 \%$ respectively) in the treatment $\mathrm{T} 1$ i.e., N1 (20:40:20 NPK).

\section{References}

Anonymous. 2010-2011. pasted economics, Generic crop India IIPR English, Production and Productivity. Pulses Development Scheme, ZPD, Kanpur.

Asthana, A.N. and Masood Ali Eds. Indian Society of Pulses Research and Development, IIPR, Kanpur, India. pp. 475-487.

Awomi, T., Alben, Singh, A.K., Kumar, Manoj and Bordoloi, L.J. 2012. Effect of phosphorus, molybdenum and cobalt nutrition on yield and quality of mungbean (Vigna radiata L.) in Acidic
Soil of Northeast India. Indian J. Hill Farming, 25(2): 22-26.

Dutta, R.K., M. Uddin and L. Rahman. 1984. Productivity of mungbean, rice and mustard in relation to boron in Brahmaputra.

Kaisher, M.S., M.T. Rahman, M.H.H. Amin, A.S.M., Amanullah, A.S.M. Ahsanullah. 2010. Effects of sulphur and boron on the seed yield and protein content of mungbean. Bangladesh Res. Publ. J., 3(4): 1181-1186.

Mondal, S.S., A. Ghosh, S. Biswajit, D. Acharya and B. Sarkar. 2003. Studies on the effect of potassium, sulphur and irrigation on growth and yield of greengram. J. Interacademicia, 7: 273277.

Saha, A., B.K. Mandal and P. Mukhopadyay. 1996. Residual effect of boron and molybdenum on the yield of succeeding mungbean in summer. Indian Agriculturist, 40: 11-16.

Sharma, P. and Khurana, A.S. 1997. Biofertilizers, Farmers and Parliament, pp. 17-18.

Tisdale, S.L., Nelson, W.L., Beaton, J.D. and Havlin, J.L. 2010. Soil fertility and fertilizer: An introduction to nutrient management. PHI Learning Pvt. Ltd., New Delhi, p. 184.

Yadav, D.S., K.S. Panwar and V. K. Singh.1997. Management of pulse crops in sequential cropping. Recent Advances in Pulses Research, 1997. A.N. Asthana and Masood Ali (Eds), Indian Society of Pulses Research and Development, IIPR, Kanpur, India. pp. 475-487.

\section{How to cite this article:}

Preeti Choudhary, Gautam Ghosh, Neha and Shobha Kumari. 2017. Study the Response of Different Phosphorus Levels and Frequency of Boron Levels on Growth and Yield of Greengram. Int.J.Curr.Microbiol.App.Sci. 6(6): 875-883. doi: https://doi.org/10.20546/ijcmas.2017.606.103 